\title{
Upgrading and testing the 3D reconstruction of gamma-ray air showers as observed with an array of Imaging Atmospheric Cherenkov telescopes
}

\author{
M. Naumann-Godó, ${ }^{\mathrm{a}}$, M. Lemoine-Goumard ${ }^{\mathrm{b}}$, B. Degrange ${ }^{\mathrm{a}}$ \\ ${ }^{a}$ Laboratoire Leprince-Ringuet, École Polytechnique, IN2P3/CNRS, F 91128 Palaiseau Cedex, France \\ ${ }^{b}$ Centre d'Études Nucléaires de Bordeaux-Gradignan, Chemin du solarium, BP 120, F 33175 Gradignan Cedex, France
}

\begin{abstract}
Stereoscopic arrays of Imaging Atmospheric Cherenkov Telescopes allow to reconstruct gammaray-induced showers in 3 dimensions, which offers several advantages: direct access to the shower parameters in space and straightforward calorimetric measurement of the incident energy. In addition, correlations between the different images of the same shower are taken into account. An analysis method based on a simple 3D-model of electromagnetic showers was recently implemented in the framework of the H.E.S.S. experiment. In the present article, the method is completed by an additional quality criterion, which reduces the background contamination by a factor of about 2 in the case of extended sources, while keeping gamma-ray efficiency at a high level. On the other hand, the dramatic flares of the blazar PKS 2155-304 in July 2006, which provided H.E.S.S. data with an almost pure gamma-ray sample, offered the unique opportunity of a precision test of the 3D-reconstruction method as well as of the H.E.S.S. simulations used in its calibration. An agreement at a few percent level is found between data and simulations for the distributions of all 3D shower parameters.
\end{abstract}

Key words: Gamma-ray astronomy, H.E.S.S., Stereoscopy, Cherenkov telescopes, 3D-reconstruction, Analysis method PACS: 95.55.Ka, 95.75.-z

\section{Introduction}

During the last four years, an important gain in sensitivity was achieved in the very-highenergy domain of gamma-ray astronomy, resulting in an increase of the number of confirmed sources by a factor of about ten. This progress was made possible by the new generation of Imaging Atmospheric Cherenkov Telescopes, and particularly by stereoscopic arrays of such detectors. Presently and in the near future, the number of individual telescopes in the different arrays is limited: 5 in H.E.S.S.-II [1], 4 in CANGAROO-3 [2], H.E.S.S.-I [3] and VERITAS [4] and 2 in MAGIC-II [5]. In the long term, much larger arrays are considered in order to improve both the sensitivity (through the rejection of hadronic showers and the increase of the

Email address: naumann-godo@llr.in2p3.fr (M. Naumann-Godó) 
effective detection area) and the angular resolution. The 3D-reconstruction of gamma-ray showers developed by M. Lemoine-Goumard et al. [6] in the framework of the H.E.S.S. experiment is particularly well suited to large arrays since correlations between different stereoscopic views of the same shower are taken into account. Furthermore, in this method, gamma-rays are selected on the basis of physical properties: the rotational symmetry of an electromagnetic shower and the lateral spread of the corresponding Cherenkov photosphere at shower maximum. The total number of Cherenkov photons emitted by the shower is then reconstructed, yielding the gammaray energy in a straightforward way. This 3D-model was successfully applied in some H.E.S.S. studies, both on point-like sources (e.g. the blazar H2356-309 [9]) and on extended sources (e.g. the supernova remnant RX J0852.0-4622 [10], also named Vela Junior).

In the present article, we first describe a significant improvement in the model which has been achieved since the original publication [6]. In the 3D-reconstruction method, the shower parameters are obtained by a maximum likelihood fit to the charge contents of the pixels of each camera, with the single constraint of rotational symmetry 1. However, in the version described in [6], no goodness-of-fit parameters were used in the further selection of gamma-ray showers. This conservative strategy, in which only a loose convergence criterion was required, was due to the fact that light fluctuations at the pixel level were not perfectly controlled and that correlations between the contents of different pixels were not taken into account. Moreover, in order to be reliable, a goodness-of-fit parameter based on individual pixel charges would require a complete modelling of the effects of the night-sky background. In section 2 below, we show that the situation is simpler at the level of each triggered telescope, and that we can directly compare the total photo-electron yield per camera predicted by the model to the actually measured one. Furthermore, in non-triggered telescopes, the model should predict a modest light yield for genuine gamma-ray showers. Such comparisons allow to define self-consistency conditions which should be satisfied for most electromagnetic showers but not necessarily for hadronic ones. Applying this additional quality criterion to H.E.S.S. data on an extended source 2 , the hadronic background remaining after using standard 3D-cuts is reduced by a factor of about 2 , while gamma-ray efficiency is kept at a high level $(\sim 80 \%)$. The significance of a weak gamma-ray signal can thus be improved by a factor 1.24 with respect to the standard 3D-analysis.

The performance of the 3D-analysis in the H.E.S.S. experiment was estimated from gammaray simulations which were also used to calibrate the energy measurement in this method. The simulation procedure used in this study consists of two parts: (a) development of gamma-rayinduced showers in the atmosphere (program KASCADE [14]) and (b) complete description of the detection chain (program SMASH [15]), namely optics, triggering electronics and readout electronics of the H.E.S.S. telescopes. In step (b) the average evolution of the detector with time is taken into account, since the efficiency of the whole acquisition chain (mirror reflectivity, light collectors efficiency, quantum efficiency of the phototubes, electronic charge measurement) is regularly monitored on the basis of ring-like images produced by muons [11] [12] when telescopes are triggered individually. The dramatic flares of the blazar PKS 2155-304 in July 2006 [13] in which the average intensity of the source was about 7 times that of the Crab nebula,

\footnotetext{
${ }^{1}$ The effect of the weak geomagnetic field $(20 \mu \mathrm{T}$ on the H.E.S.S. site, due to the South Atlantic Anomaly) is taken into account in the simulations and in the calculation of the performance of the method.

${ }^{2}$ In the case of an extended source, no cut on the angle between the source nominal position and the shower axis is applied.
} 

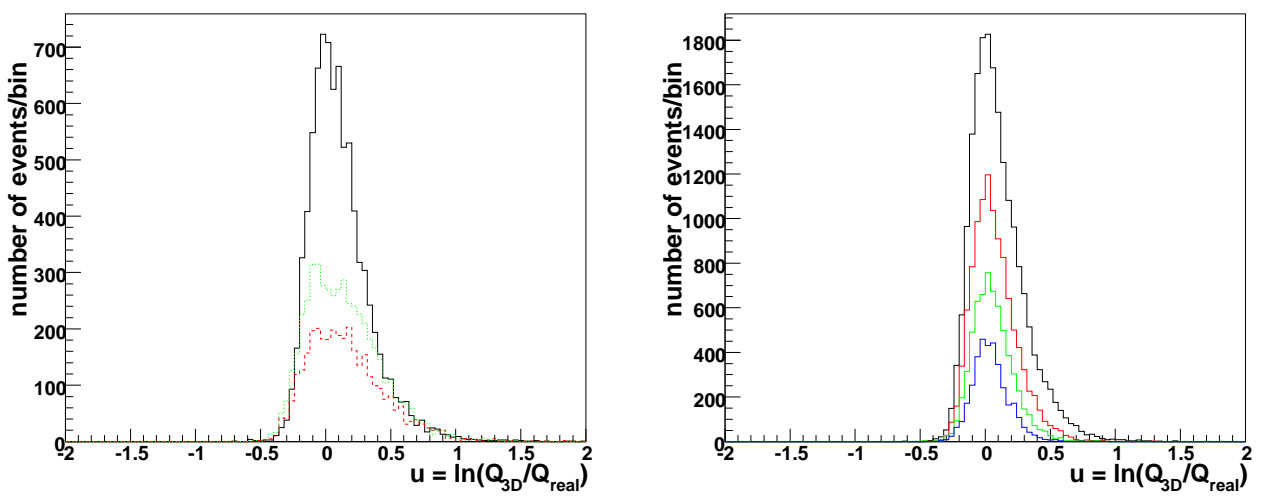

Figure 1: Left panel: distribution of the variable $u=\ln \left(Q_{3 D} / Q_{\text {real }}\right)$ for gamma-ray showers simulated at a zenith angle of $18^{\circ}$. From top to bottom: showers viewed by 2 telescopes (black), 4 telescopes (green) and 3 telescopes (red). Right panel: distribution of the variable $u=\ln \left(Q_{3 D} / Q_{\text {real }}\right)$ for gamma-ray showers simulated at different zenith angles $\zeta$ and viewed by 2 telescopes. From top to bottom: $\zeta=18^{\circ}, 37^{\circ}, 48^{\circ}$ and $53^{\circ}$. Gamma-rays were simulated according to a power-law energy spectrum with a spectral index of 2.2 .

provided us with a gamma-ray beam with a very small contamination of hadronic background, offering a unique opportunity of checking both the performance of the 3D-model and the quality of the whole simulation chain with a high accuracy. The corresponding tests are described in section 3

\section{Characterising the quality of the likelihood fit}

The 3D reconstruction method is based on a likelihood fit in which the shower parameters in space are obtained from the charge contents (in single photo-electron units, hereafter p.e.) of individual camera pixels (see formula (1) of reference [6]). Images were previously submitted to a cleaning procedure in order to remove isolated clusters of pixels with a small charge which likely result from the night sky background. The likelihood fit was restricted to those pixels retained by the cleaning procedure and to their immediate cleared neighbours, as explained in [6]. On the other hand, starting from the fitted shower parameters, it is possible to calculate the total photo-electron yield $Q_{3 D}$ expected from the 3D model in each camera. This quantity is also calculated for telescopes which were not triggered by the processed event and for which no image is available, since, in the framework of the H.E.S.S. trigger and acquisition system [7], they are not read out. It should be emphasised that, for a given triggered telescope, the predicted charge $Q_{3 D}$, as well as the measured charge $Q_{\text {real }}$ to which it will be compared, encompass the whole field of view (except for those pixels invalidated by the calibration) and not only the restricted sample of pixels used in the fit. Extending the comparison to the full field of view allows a better rejection of hadronic showers, generally more patchy than those of electromagnetic ones, and in which light is spilled over a region larger than the image core. 

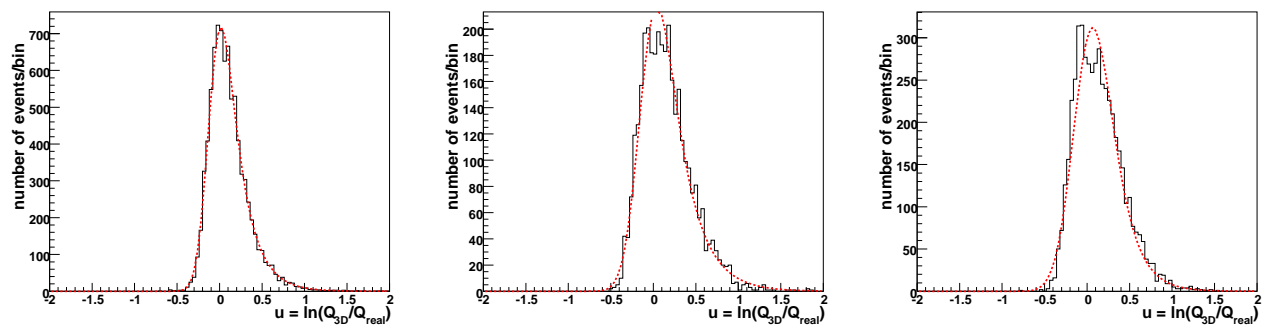

Figure 2: Distributions of the variable $u=\ln \left(Q_{3 D} / Q_{\text {real }}\right)$ for gamma-ray showers simulated at a zenith angle of $18^{\circ}$ and viewed by 2, 3 and 4 telescopes (from left to right). Dotted lines show the distributions fitted according to formula (1).

\subsection{Quality criterion on images from triggered telescopes}

The compatibility between the model and the image can be characterised by the variable $u=\ln \left(Q_{3 D} / Q_{\text {real }}\right)$. Simulations of gamma-ray-induced showers at different energies and zenith angles were used to find the expected $u$ distributions. In these simulations, the complete detection chain is taken into account. Simulated images were further submitted to the usual analysis procedure based on the 3D-model. The distribution of the variable $u$ is shown in Fig. 11 (left panel) for gamma-ray showers simulated at a zenith angle of $18^{\circ}$, according to a power-law energy spectrum with a spectral index of 2.2. Using different simulated spectra (e.g. with spectral indices greater than 3) does not significantly change the distribution. This will be further illustrated in section 3 on real data from the blazar PKS 2155-304 whose spectral index is 3.3 (see Fig. 13). The distribution peaks at $u=0$ as expected and is slightly asymmetric as shown in Fig. 1, right panel, for events triggering 2 telescopes at different zenith angles. Fig. 1 (left panel) also shows some dependence on the telescope multiplicity, i.e. the number $n_{T}$ of triggered telescopes. The difference between the case $n_{T}=2$ and that of higher multiplicities is related to the property of rotational symmetry of the shower which is assumed but not really checked if $n_{T}=2$, whereas it acts as an additional constraint for $n_{T} \geq 3$, resulting in a slightly broader distribution of the variable $u$ in the latter case.

For $u<0$ the distribution is well described by a Gaussian, whereas it behaves as an exponential one for large positive values of $u$. Therefore the probability density function $p(u)$ can be conveniently described by the convolution of a Gaussian distribution, with mean value $u_{0}$ and r.m.s. $s$, and of an exponential one with mean value $1 / \lambda$, namely :

$$
p(u)=K e^{-\lambda u} \text { freq }\left(\frac{u-u_{0}}{s}\right) \text { with } \operatorname{freq}(x)=\frac{1}{\sqrt{2 \pi}} \int_{-\infty}^{x} e^{-u^{2} / 2} d u
$$

the normalization factor $K$ being given by :

$$
K=\lambda \exp \left(\lambda u_{0}-\frac{\lambda^{2} s^{2}}{2}\right)
$$

The parameters $u_{0}, s$ and $\lambda$ were fitted to the histograms obtained from simulations. The corresponding fitted distributions are indicated by the curves superimposed to the histograms in Fig. 2 The interest of the analytic parametrisation (1) is that the corresponding cumulative distribution 

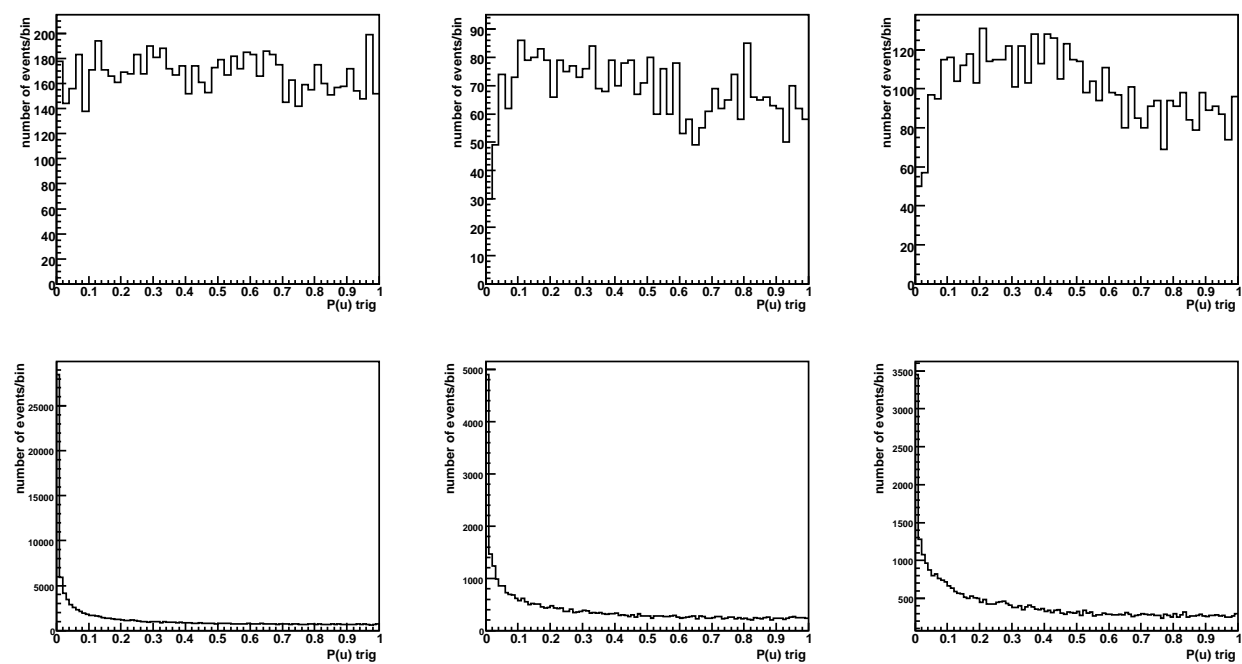

Figure 3: Distributions of the goodness variable $P(u)$ for showers viewed by 2, 3 and 4 telescopes (from left to right). Top: gamma-ray showers simulated at a zenith angle of $18^{\circ}$, according to a power-law energy spectrum with a spectral index of 2.2. Bottom: hadronic showers as observed by H.E.S.S. at a zenith angle of $20^{\circ}$ in a field of view with no significant gamma-ray emission.

is easily calculated as :

$$
Q(u)=\int_{-\infty}^{u} p(v) d v=\text { freq }\left(\frac{u-u_{0}+\lambda s^{2}}{s}\right)-\frac{p(u)}{\lambda}
$$

The distribution of $Q(u)$ is thus expected to be uniform for gamma-ray showers, whereas hadronic ones will exhibit peaks for small values of $Q(u)$ (corresponding to strongly negative values of $u$ ) and for large values (corresponding to strongly positive values of $u$ ). It is convenient to fold this distribution in order to obtain a variable whose behaviour is the same as a $\chi^{2}$ probability. This is done by using the following variable :

$$
P(u)=2 \min (Q(u), 1-Q(u))
$$

$P(u)$ will be called a "goodness variable" in the following. The distributions of $P(u)$ for those simulated gamma-ray showers referred to in Fig. 1 (left panel) are shown in Fig. 3 (top panels) and are fairly uniform 3 . On the other hand, distributions of $P(u)$ for hadronic showers detected by H.E.S.S. at a zenith angle of $20^{\circ}$ in a field of view with no significant gamma-ray emission are shown in Fig. 3 (bottom panels) for telescope multiplicities $n_{T}=2,3$ and 4 and show a strong accumulation at very low values.

In practice, the dependence of the parameters $u_{0}, s$ and $\lambda$ as functions of the telescope multiplicity $n_{T}$ and the zenith angle $\zeta$ is determined on the basis of simulations. The parameter $u_{0}$

\footnotetext{
${ }^{3}$ Of course, these distributions need not be exactly uniform, the effect of the cuts on $P(u)$ being determined by simulations.
} 


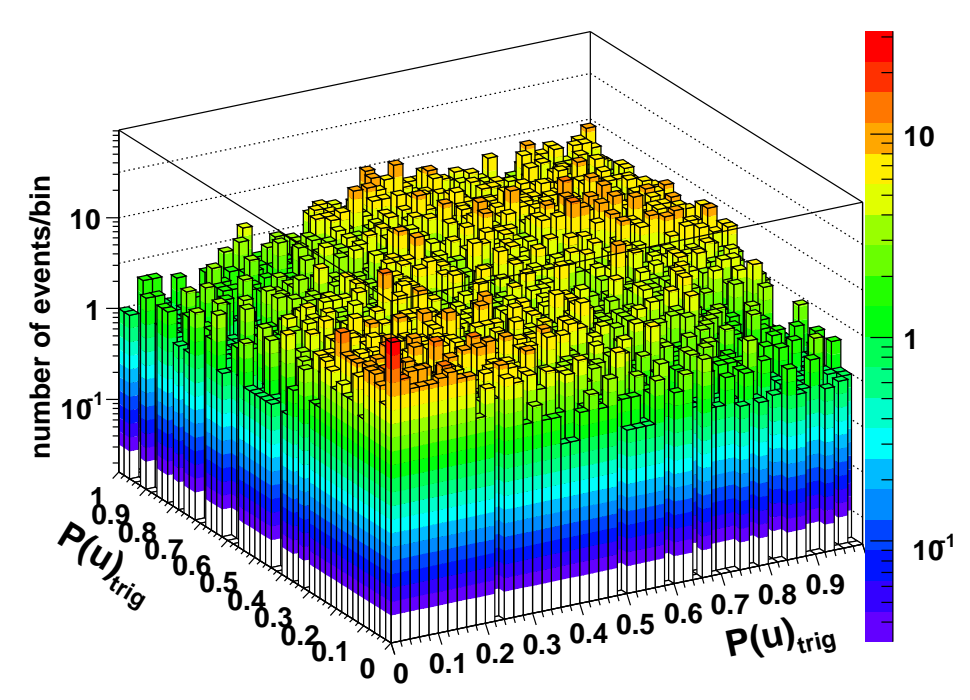

Figure 4: Gamma-ray showers simulated at a zenith angle of $18^{\circ}:$ the goodness variables $P(u)$ of the same event viewed by two telescopes are plotted versus each other.

is always found to be compatible with 0 within the statistical errors, which confirms the quality of the reconstruction method. As far as the other two parameters $s$ and $\lambda$ are concerned, the empirical formulae shown in Table 1 obtained from simulations, give a satisfactory description of the $u$ distributions in all conditions of observation.

\begin{tabular}{|c|c|c|}
\hline$n_{T}$ & $s$ & $\lambda$ \\
\hline 2 & $0.183-0.186 \cos \zeta+0.134 \cos ^{2} \zeta$ & $\min (6.3,9.358-4.411 \cos \zeta)$ \\
3 & $0.084+0.087 \cos \zeta$ & $7.141-3.528 \cos \zeta$ \\
4 & $0.108+0.062 \cos \zeta$ & $8.703-4.986 \cos \zeta$ \\
\hline
\end{tabular}

Table 1: Formulae giving the parameters $s$ and $\lambda$ defined in equation 1 as functions of the telescope multiplicity $n_{T}$ and of the zenith angle $\zeta$. These formulae were obtained from simulations.

For each event, formulae (1), (2) and (3) and those given in Table 1 allow to calculate one goodness variable $P(u)$ per triggered telescope. For a given event, these quantities are correlated ; in particular, in the case of genuine gamma-ray showers, there is a clear positive correlation between the different values of $P(u)$. Therefore, if $P(u)$ is required to be greater than a minimal value $p_{\min }$ for all triggered telescopes, the gamma-ray selection efficiency of such a cut is greater than $p_{\min }^{n_{T}}$. This is verified from Fig. 4 in which two values of $P(u)$ of each gamma-ray shower are plotted versus each other. The regions close to the axes are depopulated with respect to that surrounding the diagonal. The slight excess close to the origin (about 20 events for a total of 8400 simulated gamma-rays) is compatible with the contribution of hadron photo-production in the primary interaction (occurring with a probability of $\approx 2.8 \times 10^{-3}[17]$ ), which is taken 


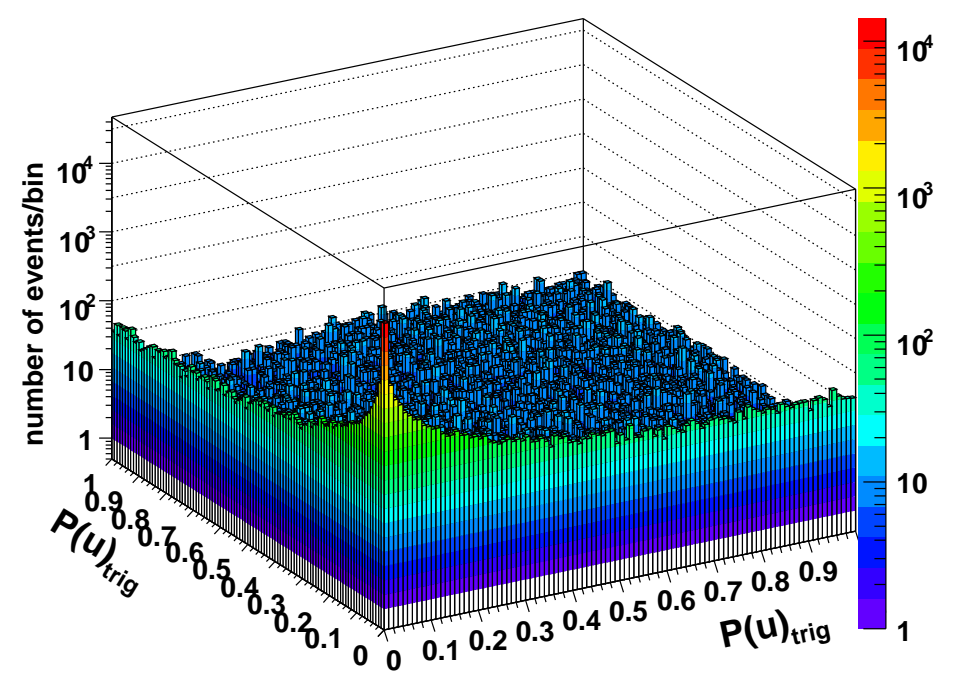

Figure 5: Hadronic showers as observed by H.E.S.S. at a zenith angle of $20^{\circ}$ in a field of view with no significant gamma-ray emission: the goodness variables $P(u)$ of the same event viewed by two telescopes are plotted versus each other.

into account in the simulation. On the other hand, the same cut provides a powerful hadronic rejection, as shown in Fig. 5 in which two values of $P(u)$ of each hadronic shower are plotted versus each other. Those events for which at least one probability is very small accumulate in two "walls" and a spectacular spike is visible at the origin.

\subsection{Quality criterion on images from non-triggered telescopes}

\begin{tabular}{|c|c|c|c|}
\hline$n_{T}$ & $\ln Q_{0}$ & $Q_{0}$ for $\zeta=20^{\circ}$ & $\sigma_{D}$ \\
\hline 2 & $4.367-0.092 \cos \zeta$ & 72 & $0.109+0.405 \cos \zeta$ \\
3 & $\max (4.5,4.296+0.288 \cos \zeta)$ & 96 & $0.184+0.259 \cos \zeta$ \\
\hline
\end{tabular}

Table 2: Formulae giving $Q_{0}$ (in p.e.) and the standard deviation $\sigma_{D}$ of the distributions of $\ln Q_{3 D}$ as functions of the telescope multiplicity $n_{T}$ and on the zenith angle $\zeta$. These formulae were obtained from simulations.

The preceding simulations of gamma-ray showers were also used to find the distribution of the light yield expected from the 3D-model in non-triggered telescopes. The same sample of simulated gamma-ray showers as in the preceding subsection was used to find the distributions of the charge $Q_{3 D}$ predicted by the reconstruction method in non-triggered telescopes. They are shown in Fig. 6, both for $n_{T}=2$ (i.e. 2 non-triggered telescopes) and for $n_{T}=3$ (i.e. one non-triggered telescope). It can be verified that typical values of this undetected charge range between 50 and 100 p.e. Furthermore, it turns out that the distribution of $\ln \left(Q_{3 D}\right)$ is practically Gaussian (see Fig.7). 


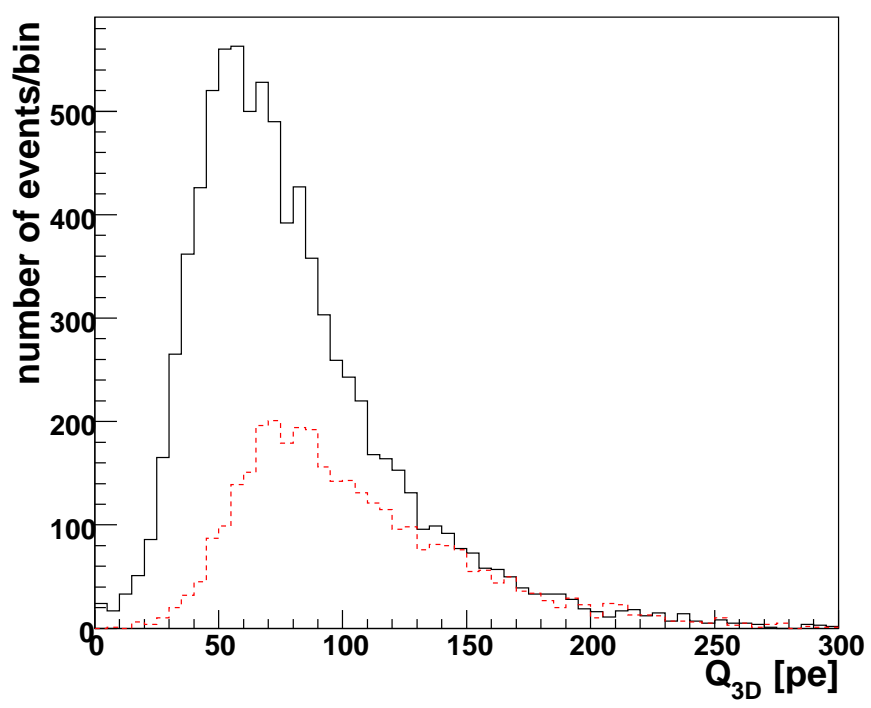

Figure 6: Distribution of the charge $Q_{3 D}$ expected from the 3D-model in non-triggered telescopes for gamma-ray showers simulated at a zenith angle of $18^{\circ}$. The solid line corresponds to events with 2 non-triggered telescopes and the dotted line to events with only one non-triggered telescope. Gamma-rays were simulated according to a power-law energy spectrum with a spectral index of 2.2 .
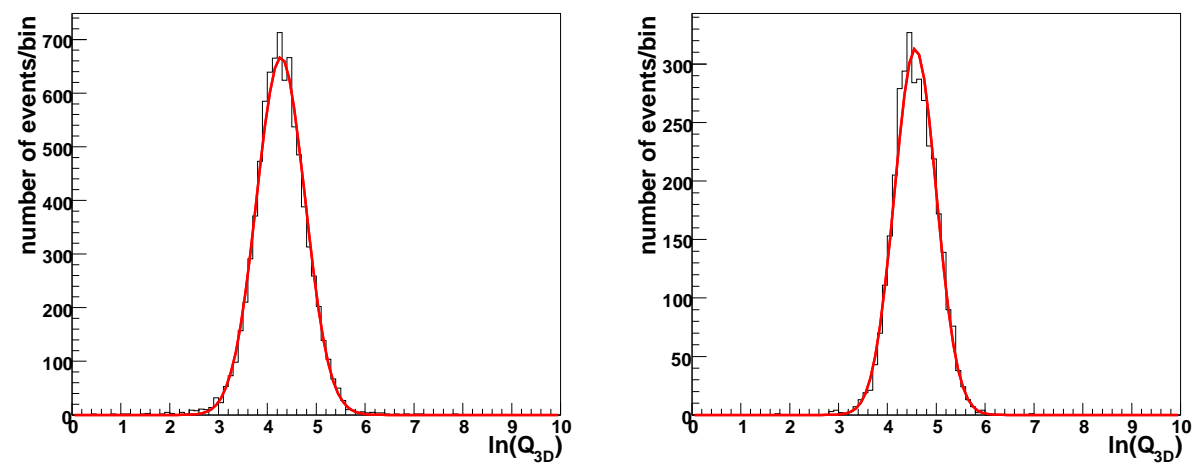

Figure 7: Distributions of $\ln \left(Q_{3 D}\right)$ for gamma-ray showers simulated at a zenith angle of $18^{\circ}$. Left panel: events with 2 non-triggered telescopes $\left(n_{T}=2\right)$ and $Q_{3 D}>20$ p.e. Right panel: events with 1 non-triggered telescope $\left(n_{T}=3\right)$. Gamma-rays were simulated according to a power-law energy spectrum with a spectral index of 2.2. 
For each zenith angle and for a given value of $n_{T}$, the average value $\ln \left(Q_{0}\right)$ and the standard deviation $\sigma_{D}$ of this distribution are determined from simulations. This allows to define the normalised Gaussian variable $v=\ln \left(Q_{3 D} / Q_{0}\right) / \sigma_{D}$, whose square $v^{2}$ behaves as a $\chi^{2}$ with one degree of freedom. It is thus possible to use the corresponding $\chi^{2}$ probability $P\left(v^{2}\right)$ as an additional goodness variable. There is one such variable per non-triggered telescope which, for gamma-ray showers, should be uniformly distributed between 0 and 1 . This is actually verified in Fig. 8 (top panels), which shows these distributions for gamma-ray showers simulated at a zenith angle of $18^{\circ}$ for $n_{T}=2$ and $n_{T}=3$. In the case of $n_{T}=2$, the small fraction $(\approx 2 \%)$ of gamma-ray events whose predicted charge $Q_{3 D}$ in a non-triggered telescope is less than 20 p.e. (see Fig. 6) has been removed from the histogram on the top left panel of Fig. 8 These events, which correspond to showers falling at large distance from the centre of the array, have a very small probability $P\left(v^{2}\right)$ and would have been rejected by the standard cuts too. There are no such events for $n_{T}=3$. Conversely, Fig. 8 (bottom panels) shows the distributions of the probability $P\left(v^{2}\right)$ for hadronic showers as observed by H.E.S.S. at a zenith angle of $20^{\circ}$ in a field of view with no significant gamma-ray emission. The accumulation at very low probabilities is very clear. The dependence of $\ln Q_{0}$ and that of the standard deviation $\sigma_{D}$ of the distributions of $\ln Q_{3 D}$ on the telescope multiplicity $n_{T}$ and on the zenith angle $\zeta$ are obtained from simulations and are given in Table 2 The values found for $Q_{0}$ are comparable to typical cutoff values for the charge collected in a given telescope, as used in other H.E.S.S. analysis methods [16 [18].

\subsection{Overall quality criterion and performance of the method}

For any event reconstructed by the 3D-model, 4 goodness variables are available, one for each telescope, whatever the trigger decision. For gamma-ray showers, these quantities should be uniformly distributed between 0 and 1 , whereas hadronic ones should most of the time lead to low values. The new quality criterion requires the goodness variables of all triggered telescopes to be higher than $p_{\text {trig }}=0.05$ and the goodness variables of all non-triggered telescopes to be higher than $p_{\text {dark }}=0.07$. This ensures that the data are compatible with the basic assumptions of the model (mainly rotational symmetry) and with the fitted values of the shower parameters. After this first step, the standard selection cuts described in [6] are applied. They require the compatibility of the fitted parameters with those of an electromagnetic shower, namely :

- compatibility between the depth of shower maximum and the number of Cherenkov photons (formula (2) of reference [6]);

- restriction on the shower 3D-width, in practice on the "reduced 3D-width", whose distribution is almost independent of the zenith angle (formula (3) of reference [6]).

We have checked that the distributions of the different goodness variables defined above did not change significantly after applying the two preceding standard cuts. Finally, in the case of a point-like source, a cut is applied on the angle $\theta$ between the reconstructed direction and that of the source [6], namely $\theta<0.1^{\circ} / \cos \zeta$.

The new method is compared to the standard 3D analysi 4 in Table 3 for an extended source (i.e. without any cut on the shower direction) and in Table 4 for a point-like source. For each kind of analysis, gamma-ray selection efficiencies $\epsilon$ are obtained from simulations assuming a

${ }^{4}$ Compared to the H.E.S.S. standard analysis, based on the mean-scaled widths and lengths of shower images, the standard 3D analysis provides a gain of 1.12 in significance [9]. 

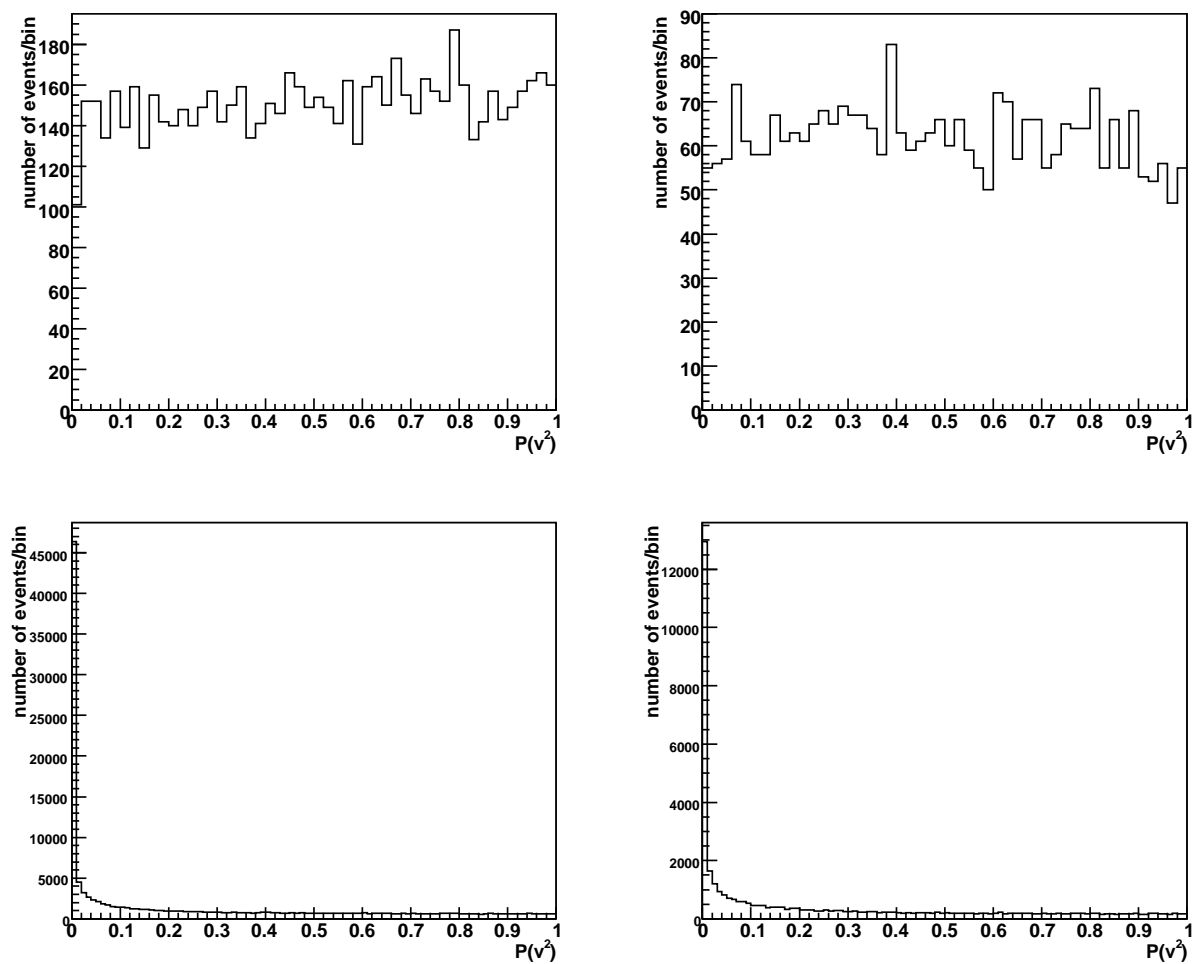

Figure 8: Distributions of the probability $P\left(v^{2}\right)$ obtained from non-triggered telescopes for events with 2 non-triggered telescopes $\left(n_{T}=2\right.$, left panels) and events with 1 non-triggered telescope $\left(n_{T}=3\right.$, right panel). Top panels: gamma-ray showers simulated at a zenith angle of $18^{\circ}$, according to a power-law energy spectrum with a spectral index of 2.2 . Bottom panels: hadronic showers as observed by H.E.S.S. at a zenith angle of $20^{\circ}$ in a field of view with no significant gamma-ray emission.

\begin{tabular}{|cc||c|c|c|}
\hline \multicolumn{2}{|c||}{ Zenith angle } & $18^{\circ}$ & $37^{\circ}$ & $46^{\circ}$ \\
\hline \hline 3D & $\epsilon_{g}$ & 0.92 & 0.91 & 0.91 \\
standard & $R_{h}$ & 9.95 & 9.69 & 10.51 \\
analysis & $Q_{\text {ext }}$ & 2.89 & 2.85 & 2.94 \\
\hline \hline New & $\epsilon_{g}$ & 0.78 & 0.78 & 0.77 \\
3D & $R_{h}$ & 21.01 & 18.54 & 18.05 \\
analysis & $Q_{\text {ext }}$ & 3.59 & 3.34 & 3.26 \\
& Gain $_{\text {ext }}$ & 1.24 & 1.17 & 1.11 \\
\hline
\end{tabular}

Table 3: New 3D analysis as compared to the standard one for an extended source (no angular cut is applied): $\epsilon_{g}$ is the gamma-ray selection efficiency, $R_{h}$ the hadronic rejection factor and $Q_{\mathrm{ext}}=\epsilon_{g} \sqrt{R_{h}}$ the quality factor. The gain in significance $\left(\right.$ Gain $_{\mathrm{ext}}$ ) is given by the ratio of quality factors. 


\begin{tabular}{|cc||c|c|c|}
\hline \multicolumn{2}{|c||}{ Zenith angle } & $18^{\circ}$ & $37^{\circ}$ & $46^{\circ}$ \\
\hline \hline 3D & $\epsilon_{g}$ & 0.58 & 0.60 & 0.58 \\
standard & $R_{h}$ & 5292 & 4233 & 2966 \\
analysis & $Q_{\text {point }}$ & 41.9 & 38.71 & 31.82 \\
\hline \hline New & $\epsilon_{g}$ & 0.51 & 0.52 & 0.51 \\
3D & $R_{h}$ & 8468 & 6124 & 4029 \\
analysis & $Q_{\text {point }}$ & 47.11 & 40.92 & 32.21 \\
& Gain & 1.12 & 1.06 & 1.01 \\
\hline
\end{tabular}

Table 4: New 3D analysis as compared to the standard one for a point-like source (angular cut $\theta<0.1^{\circ} / \cos \zeta$ ): $\epsilon_{g}$ is the gamma-ray selection efficiency, $R_{h}$ the hadronic rejection factor and $Q_{\text {point }}=\epsilon_{g} \sqrt{R_{h}}$ the quality factor. The gain in significance ( Gain $_{\text {point }}$ ) is given by the ratio of quality factors.

\begin{tabular}{|c|c||c|c|c|}
\hline 3D standard & Zenith angle & $18^{\circ}$ & $33^{\circ}$ & $51^{\circ}$ \\
\cline { 2 - 5 } analysis with & $R_{h}$ (extended) & 16.1 & 13.9 & 11.3 \\
$n_{T} \geq 3$ & $R_{h}$ (point-like) & 6870 & 4980 & 2170 \\
\hline
\end{tabular}

Table 5: Hadronic rejection factors for extended and point-like sources in the $3 D$ standard analysis in which the restriction $n_{T} \geq 3$ is applied. Results are reproduced from [6] which uses slightly different values of the zenith angle. For the sake of comparison with Tables 3 and 4 linear interpolations in $\cos \zeta$ yield $R_{h}$ (extended) $=13.7$ for $\zeta=37^{\circ}$ and 12.1 for $\zeta=46^{\circ}$. Similarly, $R_{h}$ (point-like $)=4560$ for $\zeta=37^{\circ}$ and 3050 for $\zeta=46^{\circ}$.

power-law energy spectrum with photon index 2.2. The loss of genuine gamma-ray events due to the new criteria ( $\sim 15 \%$ on average) is almost energy-independent above $100 \mathrm{GeV}$. The corresponding rejection factors $R$ for hadrons (i.e. the factors by which the numbers of hadronic events at the trigger level are reduced after applying selection cuts) are obtained from real H.E.S.S. data taken in fields of view with no significant gamma-ray emission. In the case of a weak source, the significance of the signal is proportional to the quality factor $Q=\epsilon \sqrt{R}$ which therefore characterises the sensitivity of the method. The ratio of the quality factors in the new 3D analysis and in the standard one respectively indicates the gain in significance obtained from the new method for a source detection. When values of $p_{\text {trig }}$ and $p_{\text {dark }}$ lower than those given above are used, the quality factors and the gain are found to be rather stable. The values chosen for these two probabilities have the advantage to significantly reduce the residual contamination of the selected sample. For an extended source at low zenith angles, the background is reduced by a factor of 2, which is important for morphological as well as for spectral studies. The gain in sensitivity, namely 1.24 at low zenith angles for an extended source, corresponds to that of an observation time increased by $50 \%$ in the framework of the standard 3D analysis. For point-like sources at low zenith angles, the gain is lower albeit still significant and the background is reduced by a factor 1.6. It should be noted that the improvement of the method is stronger for events triggering two telescopes which are the more numerous. The standard 3D analysis had sometimes been applied with the restriction $n_{T} \geq 3$ in order to achieve a higher sensitivity (see e.g. [9]). With the new analysis, the hadronic rejection factors obtained without any cut on the telescope multiplicity are greater than or comparable to those obtained in the standard 3D method with $n_{T} \geq 3$. From Table 5, in which the corresponding factors are reproduced from reference [6], it can be verified that the rejection factors obtained in the new analysis with $n_{T} \geq 2$ are higher by 20 to $30 \%$. 


\section{Testing the method and the simulations with the almost pure gamma-ray sample from the flaring period of the blazar PKS 2155-304 in July 2006}

The exceptional VHE gamma-ray flare of PKS 2155-304 during the nights MJD 53944 and MJD 53946, in which the average intensity of the outburst was about 7 times the flux observed from the Crab Nebula [13], provided us with an unprecedented data-set taken under uniform experimental conditions. The very high quality data featuring an abundance of gamma-rays with a very small contamination (less than 3\%) of hadronic background effectively provides us with a gamma-ray test-beam . To correct for these remaining hadronic events, the reflected background model subtraction method [8] has been applied. In this case, the OFF region from which the background is estimated is the exact symmetric counterpart of the $\mathrm{ON}$ region with respect to the telescope axis, so that acceptances in both regions are the same.

The simulations used for the comparison were custom-made to fit the actual experimental conditions during the observation period, such as the night sky background rate $(40 \mathrm{MHz})$ and the optical efficiency of the telescopes, which is directly measured via images of muon rings [12]. The latter is important for the calibration of the energy measurement, which relies on the reconstruction of the number of Cherenkov photons in the shower and is therefore particularly sensitive to variations in the relative optical efficiency. The data-set has been divided into samples corresponding to given intervals of zenith angle used in the simulation and the comparison between data and simulations was carried out for all these samples. The gamma-ray energy spectrum was simulated according to a power law, using the spectral index of 3.3 fitted from the data of the two flaring nights.

Observable quantities that are characteristic of the 3D-Model and related to crucial experimental parameters have been compared systematically, thereby examining the degree of accuracy of the match. In the following, all the comparisons refer to a zenith angle of $18^{\circ}$ but they are representative for the whole range of zenith angles covered by this study. For each observable $X$, whose mean values are $\bar{X}_{d}$ (real data) and $\bar{X}_{s}$ (simulations), the relative bias $2\left(\bar{X}_{d}-\bar{X}_{s}\right) /\left(\bar{X}_{d}+\bar{X}_{s}\right)=\Delta \bar{X} / \bar{X}$ is given in Table 6. All these quantities are at most of a few percent. The half-widths half-maximum $\left(H W H M_{d}\right.$ for data, $H W H M_{s}$ for simulations) of all histograms shown in Fig. 910,11 and12 (left panel) have been calculated. Table 6shows that, for all observables, the differences $\Delta(H W H M)=H W H M_{d}-H W H M_{s}$ are smaller than the bin width.

\subsection{Trigger-related quantities}

Fig. 9 shows the agreement for trigger-related quantities. The distribution of the number of pixels hit by photons in the camera is in very good agreement with the simulation. Especially the rising edge of the distribution, which is particularly sensitive to the actual experimental conditions, such as the night sky background rate, has been reproduced by the simulation to a high degree of accuracy. A second trigger-sensitive variable is the amount of charge in the third most luminous pixel in each telescope. As the trigger condition requires a threshold of 4 photoelectrons in at least three pixels in each sector of the camera, the comparison of the charge in the third most luminous pixel allows us to scrutinise the accuracy of the trigger. 

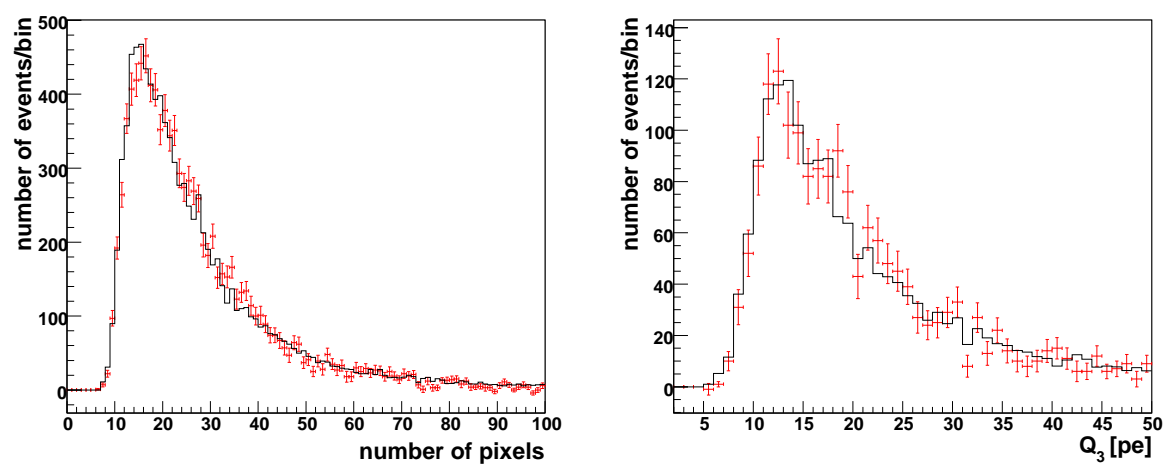

Figure 9: Comparison of observable quantities related to the trigger between the data (red points with error bars) and 3D-Model simulations (black histogram). Left panel: Number of pixels per telescope. Right panel: Charge in the third most brightest pixel of one of the telescopes.
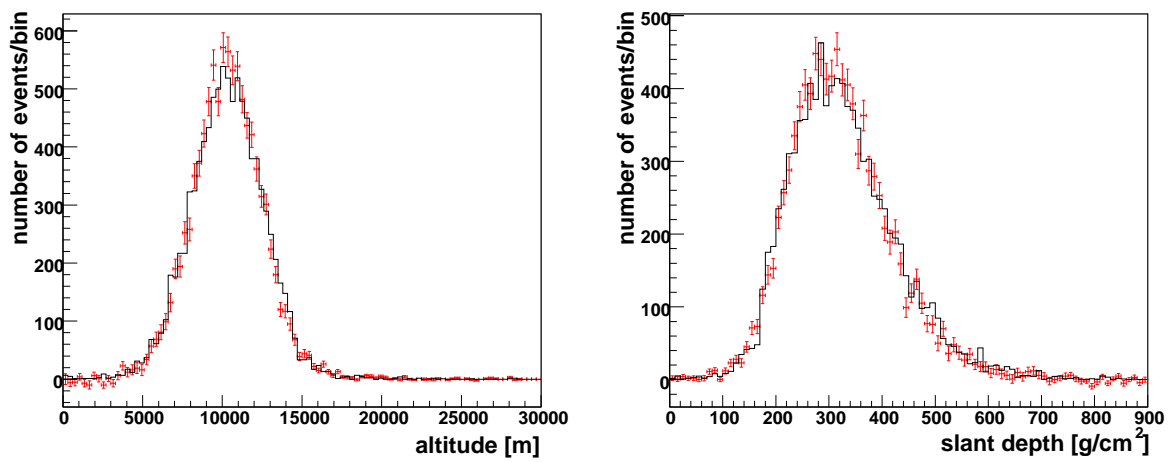

Figure 10: Comparison of observable quantities related to the atmospheric profile between the data (red points with error bars) and 3D-Model simulations (black histogram). Left panel: Altitude of the shower maximum. Right panel: Slant depth (grammage) of the shower maximum. 


\subsection{Atmospheric profile}

Other environmental conditions studied here include the atmospheric profile (desert atmosphere) used in the simulation. Whereas the altitude of shower maximum, as reconstructed by the 3D model, is a purely geometric parameter, the slant depth of shower maximum expressed in $\mathrm{g} / \mathrm{cm}^{2}$ (grammage) is determined on the basis of the atmospheric profile. Since the distributions of the first quantity show a good agreement between data and simulations (see Fig. 10, left panel), any discrepancy between the true atmospheric profile and that of simulations would induce a difference between the distributions of the slant depth obtained from simulations and data respectively. This is however not observed in Fig. 10 (right panel), which validates the atmospheric profile implemented in simulations.

\subsection{Shower characteristics}
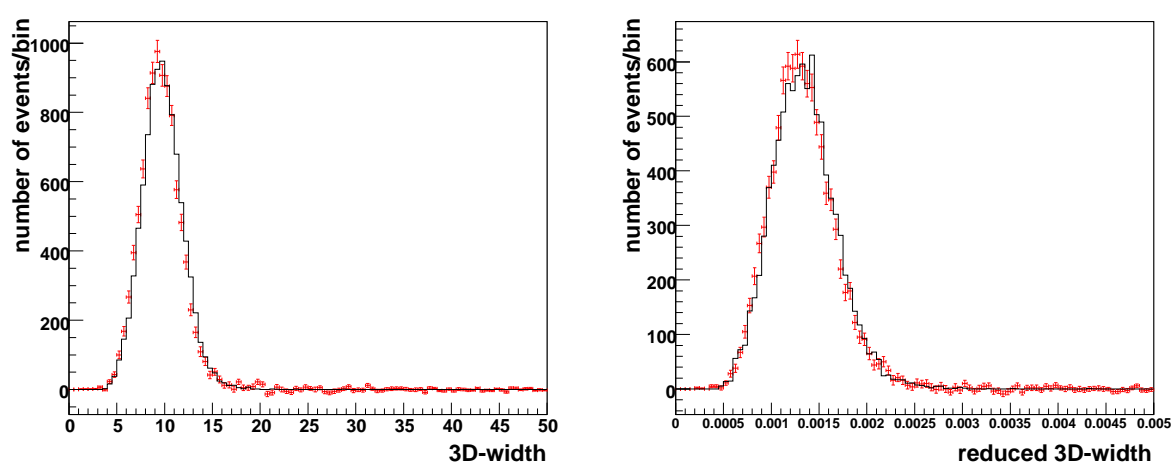

Figure 11: Comparison of observable quantities related to the atmospheric profile between the data (red points with error bars) and 3D-Model simulations (black histogram). Left panel: 3D-width. Right panel: Reduced 3D-width.

Even more pivotal than the agreement of environmental parameters is the accordance of the observable quantities upon which gamma-rays are separated from hadronic showers. In the 3Dmodel, this separation relies on the reduced 3D-width of the observed shower. Noting that the real 3D-width of the shower varies significantly with the zenith angle, a new parameter called the reduced 3D-width [6] was introduced, which is the ratio of the 3D-width to the slant depth at shower maximum, both being expressed in $\mathrm{g} / \mathrm{cm}^{2}$ at the altitude of shower maximum. This last variable was found to be almost independent of the zenith angle.

The other crucial observable quantity examined, the logarithm of the number of Cherenkov photons, is related directly to the energy measurement. As this is the single parameter in the 3D-model that has to be calibrated from simulations, its verification is essential. Fig. 12 (left panel) and Table 6 show a good agreement between data and simulations on the distribution of the logarithm of the number $N_{c}$ Cherenkov photons, when the optical efficiency derived from muon rings [12] is used in both cases. As a matter of fact, using the same optical efficiency in simulation and in data analysis is not sufficient to guarantee a good agreement between the distributions of $\ln \left(N_{c}\right)$. This is shown in Fig. 12 (right panel), in which this common efficiency has been increased by $10 \%$ with respect to that given by muon rings resulting in a manifest shift of the $\ln \left(N_{c}\right)$ distributions. Therefore, the agreement shown in Fig. 12 (left panel) and Table 6 

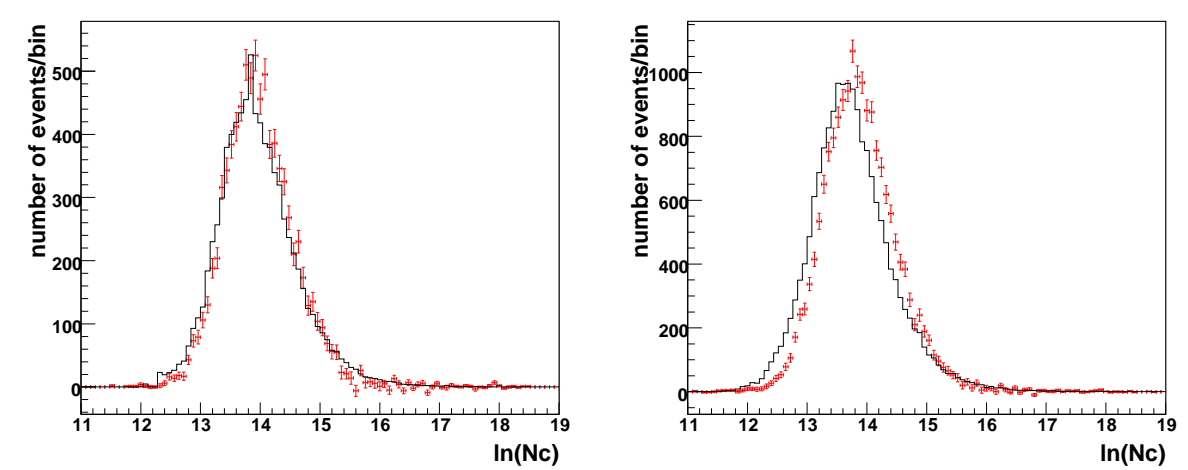

Figure 12: Comparison of the number of reconstructed Cherenkov photons for the data (red points with error bars) and 3D-Model simulations (black histograms). Left panel: the optical efficiencies have been measured from muon ring images. Right panel: the optical efficiency has been increased by $10 \%$.

implies that the optical efficiency used in the simulations (both in the event generation and in its reconstruction) matches the real one. In contrast to the previously studied variables, the energy measurement seems to be the one most affected by variations in the experimental conditions. In particular, its sensitivity to the relative optical efficiency is critical and emphasises the need for a careful calibration.

\begin{tabular}{|c||c|c|}
\hline Observable $X$ & $100 \times \frac{\Delta \bar{X}}{\bar{X}}$ & $\frac{\Delta \text { (HWHM })}{\text { Bin width }}$ \\
\hline Number of pixels per telescope & $-2.82 \pm 3.00$ & 0.62 \\
Charge in the $3^{\text {rd }}$ brightest pixel & $2.60 \pm 1.23$ & 0.17 \\
Altitude & $-0.22 \pm 0.37$ & -0.51 \\
Slant depth & $-1.11 \pm 0.55$ & -0.06 \\
3D-width & $-1.53 \pm 0.72$ & 0.31 \\
Reduced 3D-width & $-0.91 \pm 0.67$ & 0.33 \\
$\ln \left(N_{c}\right)$ & $0.18 \pm 0.10$ & -0.47 \\
\hline
\end{tabular}

Table 6: Comparison between simulated and real distributions shown in Fig. 9, 10, 11 and 12 (left panel). For each observable $X$, whose mean values are $\bar{X}_{d}$ (real data) and $\bar{X}_{s}$ (simulations), the relative bias $2\left(\bar{X}_{d}-\bar{X}_{s}\right) /\left(\bar{X}_{d}+\bar{X}_{s}\right)=\Delta \bar{X} / \bar{X}$ is given in percent units in the second column. Each distribution has also been characterised by its half-width halfmaximum $\left(H W H M_{d}\right.$ for data, $H W H M_{s}$ for simulations). In the last columnn, the difference $\Delta(H W H M)=H W H M_{d}-$ $\mathrm{HWHM}_{\mathrm{s}}$ is expressed as a fraction of the bin width.

\subsection{Quality criterion, gamma-ray selection efficiency and background rejection}

Finally, the performance of the method determined from simulations and discussed in section 2.3 can be checked on the basis of real data from the flare of PKS 2155-304. The experimental distribution of the variable $u=\ln \left(Q_{3 D} / Q_{\text {real }}\right)$ defined for triggered telescopes in section 2.1 , shown by points with error bars in Fig. 13 (left panel) for an average zenith angle of $18^{\circ}$ and 

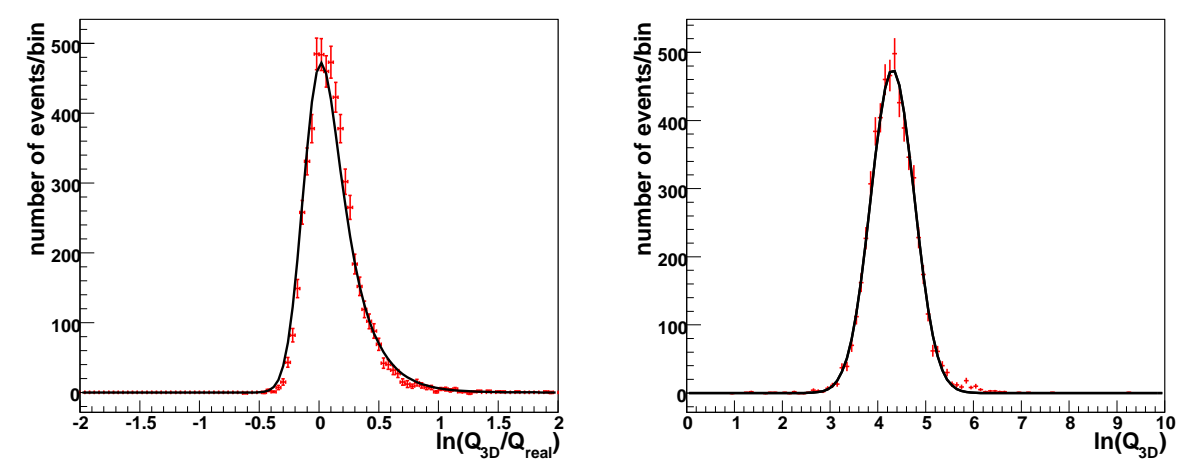

Figure 13: Left panel: comparison of the distribution of the variable $u=\ln \left(Q_{3 D} / Q_{\mathrm{real}}\right)$ for the data (points with error bars) for an average zenith angle of $18^{\circ}$ and $n_{T}=2$, to the curve deduced from formula (1) and Table 1] Right panel: comparison of the distribution of the variable $\ln \left(Q_{3 D}\right)$ for the data (points with error bars) in the same conditions, to the Gaussian distribution whose parameters are deduced from Table 2 .

$n_{T}=2$, shows a good agreement with the curve deduced from formula (1) and Table 1. In the right panel of Fig. 13, the experimental distribution of $\ln \left(Q_{3 D}\right)$ for non triggered telescopes is found to be in good agreement with the Gaussian whose parameters are deduced from Table 2 . It should be noted that both curves were obtained from simulations based on an energy spectrum with photon index 2.2, whereas the corresponding index for PKS 2155-304 during the flaring period is 3.3 [13]. This results confirms, on the basis of real data, that the distributions of the new variables $u$ and $v$ are not sensitive to the spectral index of the gamma-ray source under study, as already checked from simulations. The hadronic rejection factors at $18^{\circ}$ zenith angle obtained from PKS 2155-304 data (5085 \pm 343 for the 3D standard analysis and $7935 \pm 668$ for the new one), are found in good agreement with the figures given in Table 4 obtained from simulations for point-like sources. Only the ratio of gamma-ray selection efficiencies in the new and in the standard $3 \mathrm{D}$ analyses respectively can be checked experimentally. At $18^{\circ}$ zenith angle, this ratio, found to be $88 \%$ in PKS $2155-304$ data, is very close to the value of $87 \%$ obtained from simulations and derived from Table 4 . The preceding agreement between data and simulations, both on hadronic rejection factors and on gamma-ray selection efficiencies, validates the estimation of the gain in sensitivity achieved by the new 3D analysis given by Tables 3 and 4

\section{Conclusion}

The reconstruction method based on the 3D model of electromagnetic showers has been improved by an additional consistency check: namely, the modelled Cherenkov photosphere must reasonably reproduce the global light yield of all triggered telescopes and be compatible with a low amount of light for non-triggered ones. The new criteria involved in this consistency check are quasi-insensitive to the energy spectrum of gamma-rays used in the simulations. This was already the case for the gamma-ray/hadron discrimination criteria described in the 3D standard analysis [6] and essentially based on well-known properties of electromagnetic showers: rotational symmetry and lateral spread of the Cherenkov photosphere at shower maximum. This makes this method quite different from those based on machine learning algorithms or training 
procedures (e.g. [19]) whose sensitivity is optimised for a given gamma-ray spectrum. The gain in sensitivity with respect to the 3D standard analysis corresponds to that of an observation time increased by $50 \%$ for an extended source at low zenith angles, the hadronic background being lowered by a factor of 2 . This contamination is particularly reduced for the sample of events triggering only two telescopes, i.e. for the majority of events. Finally, the almost pure gammaray beam provided by the exceptional flare of the blazar PKS 2155-304 in July 2006 allowed to check the simulation of the H.E.S.S. experiment at the level of a few percent and to validate the gamma-ray energy measurement based on the 3D analysis. The present method, which takes account of the correlations between images observed by different telescopes and allows a rather fast processing of the events, will be particularly well suited to the exploitation of forthcoming large Cherenkov arrays.

\section{Acknowledgments}

We thank Prof. W. Hofmann, spokesman of the H.E.S.S. Collaboration and Prof. G. Fontaine, chairman of the Collaboration board, for allowing us to use H.E.S.S. data in this publication. We are grateful to Dr. M. de Naurois for carefully reading the manuscript and for providing us with very useful suggestions. Finally, our thanks go to all the members of the H.E.S.S. Collaboration for their technical support and for many stimulating discussions.

\section{References}

[1] M. Punch, in “Towards a Network of Atmospheric Cherenkov Detectors VII”, Palaiseau, France (2005), p. 379, B. Degrange et G. Fontaine ed.

[2] M. Mori, in "Towards a Network of Atmospheric Cherenkov Detectors VII", Palaiseau, France (2005), p. 19, B. Degrange et G. Fontaine ed.

[3] K. Bernlöhr, et al., Astropart. Phys. 20 (2003) 111.

[4] T.C. Weekes, in "Towards a Network of Atmospheric Cherenkov Detectors VII", Palaiseau, France (2005), p. 3, B. Degrange et G. Fontaine ed.

[5] M. Teshima, in "Towards a Network of Atmospheric Cherenkov Detectors VII”, Palaiseau, France (2005), p. 373, B. Degrange et G. Fontaine ed.

[6] M. Lemoine-Goumard, B. Degrange and M. Tluczykont, Astropart. Phys., 25 (2006) 195.

[7] S. Funk, et al., Astropart. Phys. 22 (2004) 285

[8] D. Berge, S. Funk and J. Hinton, A\&A 466 (2007) 1219.

[9] F. Aharonian, et al., A\&A 455 (2006) 461

[10] F. Aharonian, et al., ApJ 661 (2007) 236.

[11] G. Vacanti, et al., Astropart. Phys. 2 (1994) 1.

[12] F. Aharonian, et al., Astropart. Phys. 22 (2004) 109.

[13] F. Aharonian, et al., ApJ 664 (2007) L71.

[14] J. Guy, thèse de doctorat, Université Paris VI (2003); this program is a modified version of that described in M. P. Kertzman and G. H. Sembroski, Nucl. Instr. Meth. Phys. Res. A, 343 (1994) 629.

[15] J. Guy, thèse de doctorat, Université Paris VI (2003).

[16] F. Aharonian, et al., A\&A 457 (2006) 899.

[17] T.K. Gaisser, in Cosmic Rays and Particle Physics, Cambridge University Press (1990), p.245.

[18] M. de Naurois, in "Towards a Network of Atmospheric Cherenkov Detectors VII", Palaiseau, France (2005), p. 149, B. Degrange et G. Fontaine ed.

[19] S. Ohm, C. van Eldik and K. Egberts, Astropart. Phys. submitted. F. Dubois, G. Lamanna and A. Jacholkowska, Astropart. Phys. submitted. 